\title{
NEED FOR ACTION FOR A COMPANY-WIDE INTRODUCTION OF SYSTEMS ENGINEERING IN MACHINERY AND PLANT ENGINEERING
}

\author{
Wilke, Daria; \\ Schierbaum, Anja; \\ Kaiser, Lydia; \\ Dumitrescu, Roman \\ Fraunhofer Research Institute for Mechatronic Systems Design IEM
}

\begin{abstract}
Machinery and plant engineering in Germany is characterized by small and medium-sized enterprises. The so-called backbone of German industry is in transition towards Industry 4.0, with systems becoming more complex and the development task becoming an interdisciplinary task. Systems Engineering is a proven approach to realize these systems. Projects with SE approaches were accompanied and potentials of SE were structured. In this paper, we discuss the need for action for the company-wide introduction and present a solution concept.
\end{abstract}

Keywords: Systems Engineering (SE), Complexity, New product development, Organisation of product development, Mechatronics

\section{Contact:}

Wilke, Daria

Fraunhofer Research Institute for Mechatronic Systems Design IEM

Systems Engineering

Germany

daria.wilke@iem.fraunhofer.de 


\section{INTRODUCTION}

Digitization describes the networking of people and things as well as the merging of the real and virtual world through information and communication technology. It will be a major driver of innovation in the coming decades (Kagermann, 2015). It not only enables new, intelligent market services, but also offers the potential to revolutionize entire value added systems and will decisively impact the technical systems of tomorrow. The term intelligent technical systems (ITS) is also used in this context.These systems can be characterized by the following four aspects (Dumitrescu, 2013; Drossel et al., 2019):

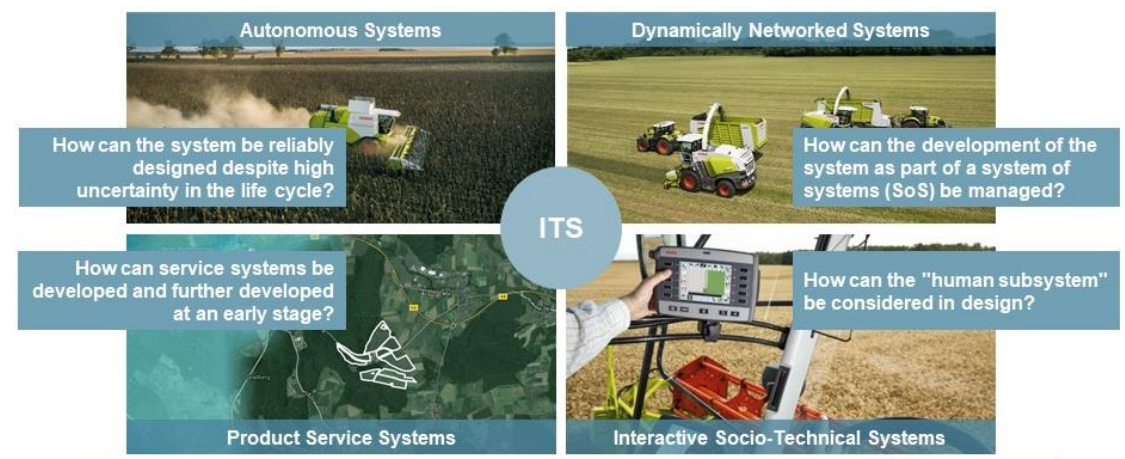

Figure 1. ITS - intelligent technical systems (Dumitrescu, 2013)

Autonomous systems solve independently complex tasks in selected application domains on the basis of functional modules of cognitive science. Without any human assistance, they learn, for example, new actions and thus react adequately to unknown events. The functionality of dynamically networked systems offers more than the sum of the functionalities of individual systems. These single systems can act autonomously from each other and can be developed independently or by different suppliers. Therefore these systems are called System-of-Systems (SoS). Customized solutions are provided by products and data-based services, which include the collection, processing and analysis of data. This results in new product-service systems. The increased interaction between human and machine results in interactive socio-technical systems: flexible adaptations to the needs of the users as well as context-sensitive support of the users by technical systems open up new possibilities.

The term system describes products (in kind), combinations of in kind and services as well as production systems as a result of a development process. The overall functionality of the systems is only revealed through the interaction of numerous subsystems; both the networking and the role of the subsystems are dynamic. The diverse relationships between the subsystems determine the overall system behavior and change continuously over the product life cycle in the sense of dynamic overall functionality.

Due to the increased interdisciplinarity as well as the higher system complexity, the product development process is becoming more complex. To master the development tasks, they require a holistic system approach and the successful orchestration of cross-domain development teams. In addition, there are further complexity drivers such as the trend towards batch size 1, data utilization over the product life cycle or the merging of product and service business. The product development of complex technical systems is a central component of manufacturing companies. Here, $85 \%$ of the later manufacturing costs of a product are already determined (International Council on Systems Engineering, 2015). The path from an initial product idea to the finished product requires the combined expertise of various disciplines along the product development process. Starting with strategic product planning, through product and service development to production system development, numerous steps are required to bring a product to series production. The successful execution of these steps is essential for the success of the company. In the machinery and plant engineering sector, the share of sales from new products was over $20 \%$ in 2015 (ZEW, 2017; Dumitrescu et al., 2018).

An approach that meets these challenges is Systems Engineering (SE) (Haberfellner et al., 2019). SE focuses on the development of holistic solutions to complex problems and considers its task to be a continuous, interdisciplinary approach to system development. All relevant design aspects, such as resilience, security, sustainability, usability, manufacturability and business model, but also aspects such as requirements, functionality, behavior and design are taken into account. It contributes 
significantly to the safe and fast development of multidisciplinary systems and claims to support the orchestration of the actors in the development of complex systems. Up to now, SE is driven by practical application and characterized by single, isolated solutions.

\section{SYSTEMS ENGINEERING IN PRACTICE}

The study "Systems Engineering in Industrial Practice" shows that SE is regarded as being consistently important in the aerospace, automotive, machinery and plant engineering sectors. Nevertheless, companies still rate their own capabilities in these areas as too low (Gausemeier et al., 2013). The demand and need for action in the individual industries increases and was confirmed in further studies (Kuhlenkötter et al., 2017; Müller et al., 2019). Many companies are undergoing a transformation and are facing up to the challenge (Dumitrescu et al., 2018). Organizational structures are being redesigned, roles and processes are being restructured, methods are being implemented and applied using pilot projects. The automotive industry is currently playing a pioneering role. Driven by scandals and the pressure to use new technologies combined with the increasing overall complexity of the systems, a wide range of activities is being pushed forward to bring about change in the development process. Original Equipment Manufacturers (OEMs) are setting up large project formats with high volumes.

Other industries and especially medium-sized enterprises (SMEs) cannot adapt this approach. They rather observe the activities and back off from these challenges. Due to their limited resources, access to and development of SE expertise is particularly difficult. Furthermore, the structure of the machinery and plant engineering industry is very heterogeneous, so that the SE needs also vary from company to company (Gausemeier et al., 2009; Bauer et al., 2018).

Despite the existing barriers, many companies have recognized the need for an interdisciplinary approach to product development. They recognize the potential to master the constantly increasing complexity of their systems and to exploit the opportunities of new markets (Gausemeier et al., 2013). It can be observed that the enterprises in the sector are only slowly approaching the subject area of SE, mainly from the development department and only partially. However, in order to increase performance and thus to achieve or secure a lead in international competition, it is necessary to establish interdisciplinary approaches in machinery and plant engineering (Schuh et al., 2017).

\subsection{ITS in the innovation cluster it's OWL}

In the north-east of North Rhine-Westphalia, a technology network, called innovation cluster it's OWL (Intelligent Technical Systems OstWestfalenLippe), is dedicated to the design of ITS by linking the key players from research and industry and pushing innovation in research projects.

OstWestfalenLippe is one of the strongest production locations in Europe and is characterized by a high concentration of employment, innovative ability and export ratio. In machinery and plant engineering, the electrical and electronics industry as well as the automotive supply industry, 400 companies provide jobs for around 80,000 employees and generate an annual turnover of 17 billion euros.

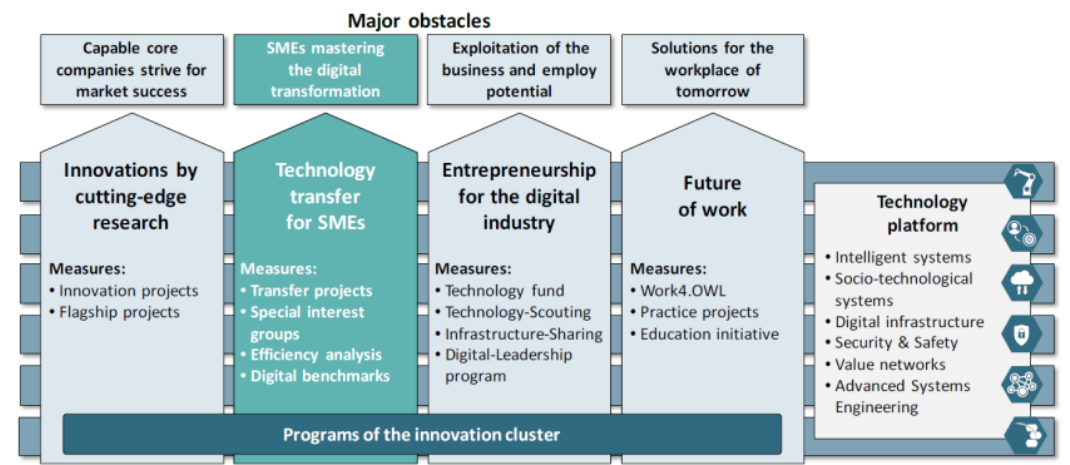

Figure 2. Structure of it's OWL (Fechtelpeter et al., 2017)

The innovation cluster is the trademark of the technology location OstWestfalenLippe. 174 enterprises, universities, research centers and organizations participate in the "Leading-Edge Cluster Strategy" in the form of an association. The spectrum of the enterprise products ranges from intelligent sensors, drives and automation components to machines, household appliances, vehicles and networked systems such as production plants, smart grids and cash management systems. The 
term Cyber-Physical Systems is used in this context. Results from the projects incorporate into the technology platform (Figure 2) and are thus available to all participating companies.

Systems Engineering has been a core topic of the technology platform from the very beginning. In a five-year research project, fundamentals of SE were developed and validated in the innovation projects (Figure 2). Thus, many enterprises in the region were familiarized with SE. However, innovation projects mainly address large, so-called core enterprises of the region. In order to make the research results accessible to all medium-sized enterprises in the cluster region as soon and effectively as possible, the Leading-Edge Cluster implemented a further construct - the SME-oriented transfer concept. This is based on a four-stage model according to KORELL/SCHAT (Korell and Schat, 2013). The steps are: (1) attention and initial information, (2) in-depth understanding, (3) trial and error, as well as (4) use and integration.

During the transfer steps different transfer formats are used. With a special transfer concept, new basic technologies from the projects are made available to small and medium-sized enterprises. A main part of the transfer program of it's OWL are tailored cooperation projects called "focused transfer projects". These cooperation between SMEs and research institutions are characterized by their application orientation and a short duration. The excellent response and feedback shows that small and mediumsized enterprises benefit in a special way from the cooperation of the network. Transfer projects give them easy access to technologies that have been proven in practice (Fechtelpeter et al., 2017).

Systems Engineering was addressed in 44 so-called focused transfer projects. For this purpose, formats were created in close cooperation between research institutions and SMEs, which have gradually focused on the challenges related to the use of SE. Together, methods were applied and tools were used.

\subsection{Analysis of completed transfer projects}

The aim of the five to ten-month transfer projects was to help SMEs in the region to achieve a higher level of technical maturity. Taking into account the specific initial situation and the individual framework conditions of the companies, the project took the first concrete steps towards the implementation of ITS. For this purpose, approaches from SE were applied. The projects were evaluated in a strict selection process.

By using SE as a instrument for the transformation of ITS in SMEs, the enterprises gained knowledge of methods and tools in practice and applied them. The fields of action in the transfer projects were manifold as well as the application varied from case to case. This resulted in many benefits for the use of SE for the enterprises, which were identified in a detailed analysis (Czaja and Dumitresu, 2015). The starting point for the analysis was the general benefit description from the literature and the results of the study "Systems Engineering in Industrial Practice" (Figure 4). These benefits do not specifically relate to the machinery and plant engineering industry (SMEs). The core of the identification of the potentials for this specific user group was the analysis of the completed transfer projects. Benefits are not exhausted possibilities which can be developed by activities of the enterprise in favour of all user groups (Schierbaum, 2020; Pümpin, 1992). For this purpose, the individual framework conditions, the concrete problem areas, the concrete approach and the concrete objectives of the individual enterprises were analyzed and thematically clustered (Figure 3). In addition to the transfer projects, results of a workshop with $\mathbf{3 0}$ experts from the field of machinery and plant engineering industry were considered. In particular, concrete problems from the enterprises have been discussed where SE can provide support. Based on this, relevant benefits were reviewed.

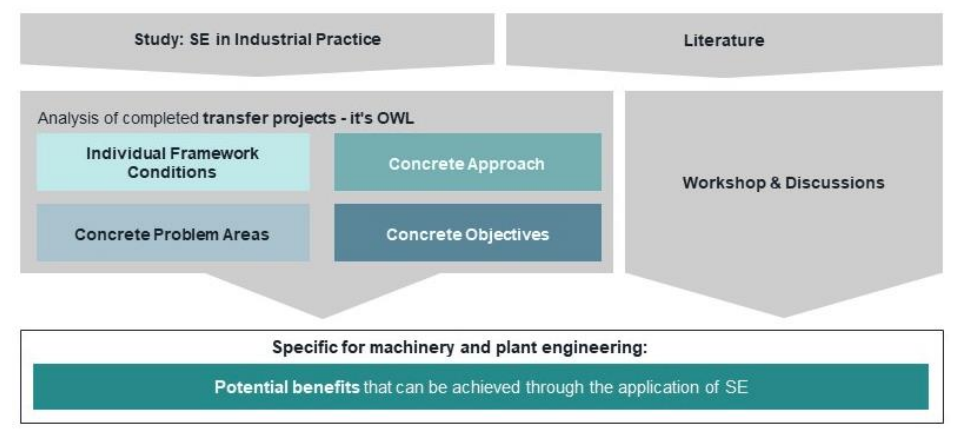

Figure 3. Analysis method to identify the benefits for medium-sized companies in machinery and plant engineering (Schierbaum, 2020) 


\subsection{Derived benefits for Systems Engineering}

Result are six significant benefits for the application of SE in small and medium-sized enterprises (Figure 4). The benefits refer to the product or market performance, the company (e.g. process and organizational structure) and the business environment (e.g. customers, value-added partners). The first four benefits can be located along the V-model of the VDI guideline 2206 and focus on the early phases of system design. The further benefits relate to the collaboration organization, both internally and across all enterprises. They focus on the aspect of project management.
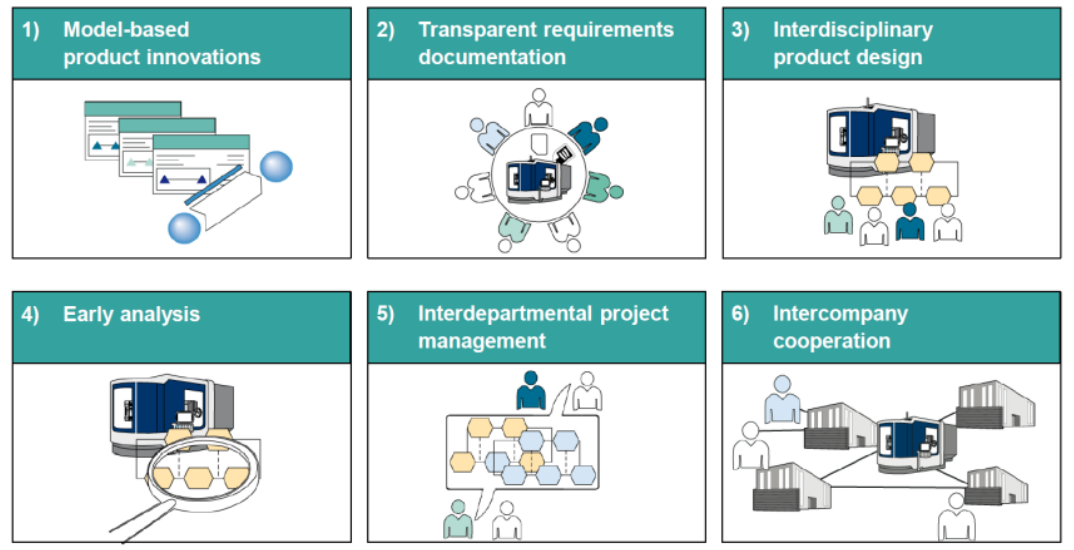

Figure 4. The six main benefits of SE in small and medium-sized enterprises (Schierbaum, 2020)

1. Model-based product innovations: The benefit is a gradual development of the market performance. Innovation and improvement potentials for products and services are identified by the enterprise. Exemplary projects have used the system model as a medium for holistic system analysis. The analysis of the current product was carried out in interdisciplinary teams. Supported by creativity methods new innovative solutions were developed.

2. Transparent requirements documentation: The benefit is based on the systematic collection of requirements as well as the consistent management. SE methods were used in the projects to identify the requirements in an interdisciplinary way. The requirements were documented and managed model-based in the system model. This procedure provides the basis for a reliable offer.

3. Interdisciplinary product design: The benefit consists in a development and description of the product concepts from the very beginning with all the technical experts involved. Interfaces are thus made transparent in the system model and interactions are taken into account at an early stage.

4. Early analysis: The benefit of a holistic analysis already in the concept phase of the product is to identify risks early, to recognize the need for requirement changes and to clarify uncertainties. With the intention of an analysis the system model was described in these projects and examined regarding certain aspects. These aspects were: changes and their systemic impacts, cost considerations, safety and security.

5. Interdepartmental project management: ITS is an interdisciplinary task that is carried out in cooperation across departmental boundaries. The management of this task was carried out in the projects based on the system model. This ensured that resources were optimally used and synchronized.

6. Intercompany cooperation: Through communication and cooperation across the enterprise, development can be systematically supported from the early development phase through the entire product creation process. This applies, for example, to communication with customers, service providers or suppliers. The system model was also used in these projects as a communication and documentation medium for interfaces and the mechatronic overall view.

The analysis of the transfer projects has also shown that certain characteristics of the enterprise, product or project reinforce a specific benefit. For this purpose, different characteristics from the areas of company, product and project were examined.

An example of a characteristic from the enterprise sector is the size of the development department. This influences, for example, the communication and cooperation of the experts involved through the use of the system model. In small development departments, which are at one location, the communication 
ways are short and face-to-face discussions are made without using the system model. From the project sector, the project size as well as the project type (new, adaptation or variant development) influences the relevance of a benefit.

\section{COMPANY-WIDE INTRODUCTION OF SYSTEMS ENGINEERING}

The introduction of SE depends on various factors. The path via benefits supports the achievement of quick added value in the enterprises. However, a company-wide steadiness cannot yet be achieved in the long term. There are many reasons for this - but the main reason is the intensive focus on SE technology (methods, language, tools). A holistic approach is necessary for successful introduction and steadiness of SE in the enterprise. For this purpose, besides the consideration of "technology", additional aspects such as "human" and "organization" must be added. ${ }^{1}$ The enterprise thus represents a socio-technical system (Figure 5):

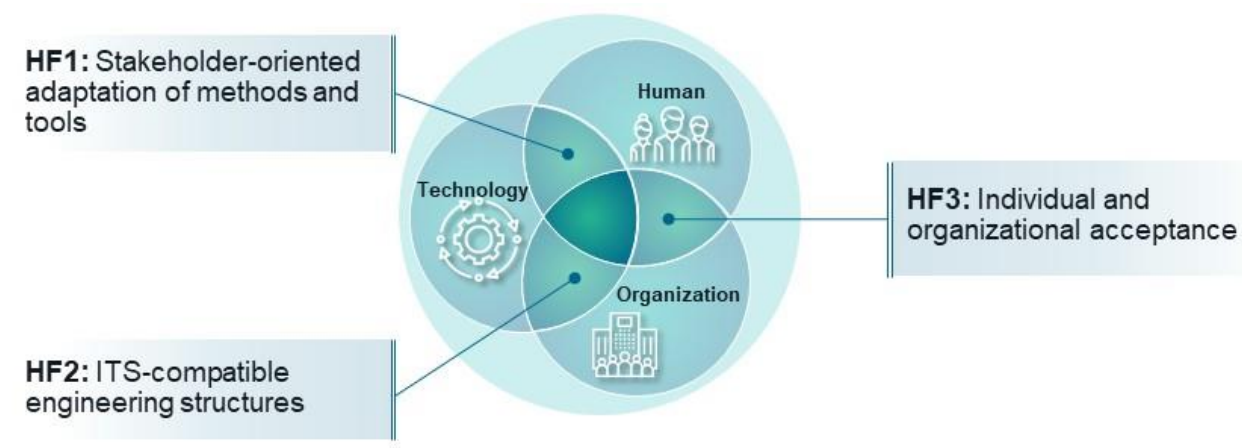

Figure 5. Human-Organization-Technology consideration

The sociotechnical system thus provides the framework for the introduction of SE. Technologies, suitable organizational structures and qualified employees are required. For a company-wide steadiness of the approaches, the attention must be focused primarily on the interfaces of the HumanOrganization-Technology consideration (Figure 5). An organization with its internal processes cannot be changed independently without considering the factors of human and technology. If a process in the company is adapted, a change process has to be undergone, which also addresses the human factor.

In addition, the benefits can only be achieved successfully in the long term by considering a holistic approach. For example, the realization of the benefit "Transparent requirements documentation" requires a technical implementation solution on the one hand. This in turn has an impact on the IT organizational structure on the other hand. Furthermore, a transparent requirements documentation also represents a new challenge for the human factor. Responsibilities must be clearly defined, as well as the authority to change the requirements documentation.

\subsection{Need for Action}

In this chapter, the interfaces to be considered are explained in more detail by describing the research questions under consideration of the factors Human-Organization-Technology (Figure 5).

Research question 1: How can methods and tools be adapted to be stakeholder-oriented?

The developed methods and tools of SE have been used and tested in many enterprises. The methods have been developed from the purely technical point of view of development activities. However, SE addresses the entire development process and the humans involved (stakeholders). The stakeholders are active in all functional areas (e.g. strategic planning, purchasing, production) and hierarchical levels (e.g. management, training) of the company. They experience the approaches as too complicated or not manageable (Gausemeier et al., 2013). The situation is similar with the corresponding tools, which were developed from the technical environment and primarily focus on supporting model creation. A sociotechnical approach is needed that gives an equal consideration to the stakeholder perspective. At an early stage, methods and tools must be considered based on stakeholder-specific needs instead of technical possibilities.

\footnotetext{
${ }^{1}$ also called "HOT Fit Model” according to DeLone and McLean (Jafari et al., 2011)
} 


\section{Research question 2: How can companies create ITS compatible engineering structures?}

The enabling of interdisciplinary cooperation requires a transformation in the organizational structure as well as a transformation of the IT systems in the engineering. With the change to ITS, enterprises are currently undergoing a transformation and, in addition to mastering the technical challenges, they have to focus more and more on organizational aspects. With ITS, interdisciplinary cooperation is becoming increasingly important. In addition to classical design, which historically has been the strongest area in the enterprises, electrical engineering and software engineering are very important. These departments in turn consist of various disciplines such as embedded software, data analytics, communication technology. To promote cooperation in the sense of the SE, both the organizational structure and the required IT systems must be reconsidered and restructured. There is a lack of holistic approaches to adapt the engineering to ITS. This complex task has to be dealt with under current situations and historically grown structures of the enterprises in the machinery and plant engineering industry.

Research question 3: How can individual and organizational acceptance be achieved in companies?

Company-wide use of SE requires acceptance on both levels: individual and organizational. Currently, there is a lack of company-wide acceptance of SE. In order to achieve this acceptance and thus ensure steadiness, approaches from change management as well as work and organizational psychology must be taken into account. These approaches provide tools to evaluate methods with regard to for instance processes, performance and presentation. The diversity and large number of stakeholders in the SE makes company-wide distribution difficult. There is a lack of approaches and learning formats that convey the breadth and depth of SE in a suitable manner for the target group.

In addition to the three fields of action resulting from consideration of the interfaces of the factors Human-Technology-Organization, a further field of action is being developed. This field of action has a superior role and addresses the transfer beyond the company borders:

Research question 4: How can the results be prepared in a manner that is as neutral as possible for SMEs in the machinery and plant engineering sector so that they can be successfully transferred to OWL?

The analysis of the benefits revealed that enterprises in the machinery and plant engineering sector all face similar or identical challenges. Together they are working on new technological solutions to master the innovative transition to ITS. These companies need to be strengthened and empowered in order to adapt their engineering to the challenges. As the solutions are distributed from medium-sized enterprises, they can be transferred to other enterprises. Transferability and acceptance can be achieved through the role model function of similar enterprises. The transformation to an SE-capable enterprise must be individually tailored. There is a lack of suitable formats and a lack of a company-neutral presentation of approaches and engineering solutions.

\subsection{Solution Approach}

These challenges lead the way for an SE-capable company. The way to get there is not trivial. Enterprises from the machinery and plant engineering sector will not be able to follow this path on their own. There is a need for research in the fields of action. In addition, the pressure to change into an SEcapable enterprise increases more and more the faster technologies change. For this reason, a new approach must be promoted that supports enterprises within the scope of research activities, as well as develops solutions close to the user and validates them in the enterprises.

This approach was chosen in a cooperative project called SE4OWL (Systems Engineering for OstWestfalenLippe) within the framework of the leading-edge cluster it's OWL. The SE4OWL project consists of three enterprises from the machinery and plant engineering sector. All three enterprises already have SE experience and contribute expertise to the project. The depth and breadth of the SE experience as well as the company-wide requirements differ greatly. This in turn reflects the complexity and challenges of the SMEs. The aim of the project is to develop a set of instruments for the companywide introduction and steadiness of Systems Engineering.

This shall be achieved by an iterative proceeding (Figure 6). For this purpose, results will be developed, validated in a company-neutral way and then tested under real conditions. Parallel to this, an abstraction will be forced, so that results will be transferred continuously and thus during the project duration and can be used by other enterprises. 


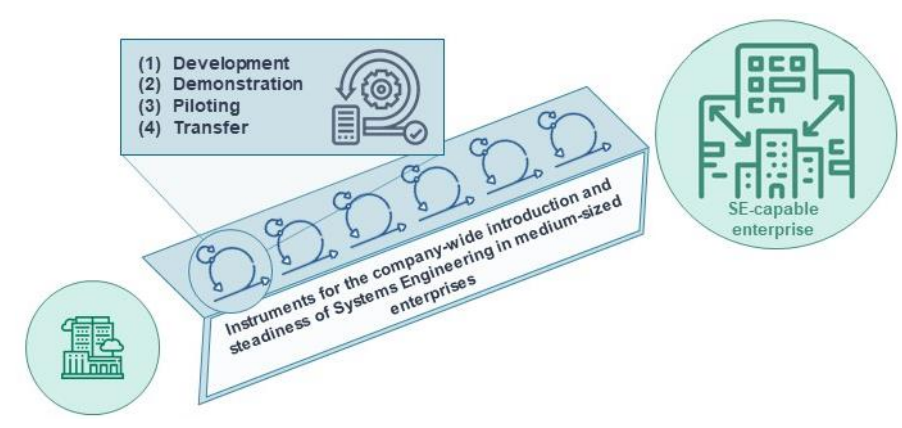

\section{Figure 6. Project structure - SE for OWL to increase the innovative ability of SMES}

In this procedure, the fields of action are addressed by defining and developing the instruments through sub-targets.

1. Scalable methods and tools: A detailed stakeholder analysis and description is carried out to adapt methods and tools to the needs of stakeholders. Methods are collected and described in a scalable way. This ensures that stakeholders can use the methods according to their purpose and needs. The associated IT systems are adapted to the methods in such a way that intelligent solutions make working with the models user-friendly.

2. Need-oriented organizational design and IT infrastructure design: For ITS-compatible engineering structures, organizational structures as well as the IT infrastructure are analyzed and adapted. The companies are enabled to develop a target vision based on the current situation. The aim is to break down the topic of SE into its components and to derive a maturity model. A methodical approach will support the enterprises to set up an introduction strategy and the implementation. Restrictions of the enterprises are to be taken into account as well as the current development of the IT systems on the market.

3. Integration and steadiness within the enterprise: For integration and steadiness within the company, SE is communicated to stakeholders using new learning methods. The needs of the stakeholders in terms of depth and breadth of content are taken into account. For this purpose, it is necessary to choose stakeholder-friendly formats, to design them and to anchor them in qualification plans of the enterprise. In addition, a communication strategy will be developed to ensure that $\mathrm{SE}$ is communicated to the right target groups.

4. Company-neutral validation and transfer to SMEs: The results will be developed companyspecifically, validated in a company-neutral way and prepared for transfer. The aim is a broad transfer to medium-sized enterprises. For this purpose, a demonstrator will be implemented that represents a fictitious enterprise. This will stereotypically represent the challenges in the engineering of ITS. The change towards an SE-capable company will be communicated by interactive solutions. The demonstrator will also serve as a medium for the neutral validation of the developed concepts.

\section{CONCLUSION}

Enterprises in the machinery and plant engineering sector are facing increasing challenges to master the transformation to Intelligent Technical Systems (ITS). The associated transformation in engineering can be achieved with the Systems Engineering (SE) approach. SE is used in many projects and benefits are targeted. However, a company-wide steadiness does not yet take place.

This paper presents a holistic solution concept that enables enterprises in the machinery and plant engineering industry to take the steps to become an SE-capable enterprise. A key factor to support SMEs is based on the socio-technical perspective. Besides addressing the technological perspective on SE, the factors human and organization have also been taken into account. This approach is structured into four research questions concerning the topics: Stakeholder-orientied adaptation of methods and tools, ITSoriented engineering structures, individual \& organizational acceptance, and transfer.

Within the framework of a project, a set of instruments for the company-wide introduction and consolidation of Systems Engineering in medium-sized enterprises is being developed. The concept provides for a project-accompanying elaboration, validation and dissemination of the SE approaches in three enterprises from the OWL region. All results will be prepared in a company-neutral manner during the project term and made available to other enterprises. The examination of the three enterprises will be 
used to describe exemplary use cases in further work. Furthermore, the case studies provide guidance for other enterprises. The innovative approach enables an efficient transfer of the results even beyond the funding phase. By embedding the project in the activities of it's OWL innovation cluster, a sustainable and broad-based exploitation of the results is ensured. At the same time, the close project-accompanying exchange within the innovation network ensures that the project has a high relevance for all stakeholders involved. The barriers previously associated with SE introduction will be lowered and easy access to the topic area will be made possible for SMEs as well.

\section{ACKNOWLEDGMENTS}

This research and development project is funded by the Ministry of Economic Affairs, Innovation, Digitalization and Energy (MWIDE) of the State of North Rhine-Westphalia within the Leading-Edge Cluster "Intelligent Technical Systems OstWestfalenLippe (it's OWL)" and managed by the Project Management Agency Jülich (PTJ). The author is responsible for the content of this publication.

\section{REFERENCES}

Bauer, W., Schlund, S., Hornung, T. and Schuler, S. (Eds.) (2018), Digitalization of industrial value chains - a review and evaluation of existing use cases of Industry 4.0 in Germany.

Czaja, A. and Dumitresu, R. (2015), Guideline for a Systems Engineering Process.

Drossel, W.-G., Ihlenfeldt, S., Langer, T. and Dumitrescu, R. (2019), “Cyber-Physical Systems”, in Neugebauer, R. (Ed.), Digital transformation, Fraunhofer-Forschungsfokus, 1st ed.

Dumitrescu, R. (Ed.) (2013), Design framework for the integration of cognitive functions into intelligent technical systems.

Dumitrescu, R., Fechtelpeter, C., Ebbersmeyer, P., Gausemeier, J., Hobscheidt, D. and Kühn, A. (2018), On the road to industry 4.0: Technology transfer in the SME sector, Paderborn.

Fechtelpeter, C., Kuehn, A., Dumitrescu, R. and Ebbesmeyer, P. (Eds.) (2017), Integrated Technology Transfer Concept For Fostering Innovation In SMEs, Vienna, Austria.

Gausemeier, J., Dumitrescu, R., Steffen, D., Tschirner, C., Czaja, A. and Wiederkehr, O. (Eds.) (2013), Systems Engineering in industrial practice, Paderborn.

Gausemeier, J., Stoll, K. and Wenzelmann, C. (2009), "Future Oriented Strategy Development”, in Proceedings of the XX ISPIM Conference, Wien.

Haberfellner, R., de Weck, O. de, Fricke, E. and Vössner, S. (2019), Systems Engineering, Springer International Publishing, Cham.

International Council on Systems Engineering (INCOSE) (Ed.) (2015), Systems Engineering Handbook: A guide for system life cycle processes and activities, 4. edition, Wiley, Hoboken, NJ.

Jafari, S.M., Noor, A.A., Murali, S. and Mohd, F.S. (2011), "A Respecification and Extension of DeLone and McLean Model of IS Success in The Citizen-Centric E-Governance”, in IEEE, pp. 342-346.

Kagermann, H. (2015), "Change Through Digitization - Value Creation in the Age of Industy 4.0", in Pinkwart, A., Albach, H., Meffert, H. and Reichwald, R. (Eds.), Management of permanent change, Springer Fachmedien Wiesbaden, Wiesbaden.

Korell, M. and Schat, h.-D. (2013), "Entwicklung eines Transfermodells”, in Warschat, J. (Ed.), Transfer von Forschungsergebnissen in die industrielle Praxis: Konzepte, Beispiele, Handlungsempfehlungen ; Zusammenfassung der Ergebnisse des Projektes Entwicklung von Transfermechanismen für die effiziente und nachhaltige Verbreitung von Forschungsergebnissen in die industrielle Praxis am Beispiel Mechatronik, Fraunhofer Verl., Stuttgart.

Kuhlenkötter, B., Wilkens, U., Bender, B., Abramovici, M., Süße, T., Göbel, J., Herzog, M., Hypki, A. and Lenkenhoff, K. (2017), New Perspectives for Generating Smart PSS Solutions - Life Cycle, Methodologies and Transformation.

Müller, P., Lindow, K., Gregorzik, S. and Stark, R. (2019), Smart industrial products. Smarte Produkte und ihr Einfluss auf Geschäftsmodelle, Zusammenarbeit, Portfolios und Infrastrukturen, Berlin.

Pümpin, C. (1992), Strategische Erfolgspositionen - Methodik der dynamischen strategischen Unternehmensführung, Bern.

Schierbaum, A. (2020), Systematik zur Ableitung bedarfsgerechter Systems Engineering Leitfäden im Maschinenbau, Paderborn.

Schuh, G., Anderl, R., Gausemeier, J., Hompel, M. ten and Wahlster, W. (Eds.) (2017), Industry 4.0 Maturity Index: Managing the digital transformation of companies, Acatech study, Herbert Utz Verlag GmbH, München.

ZEW (2017), Innovation activities of firms in Germany - Results of the German CIS 2012 and 2014:

Background report on the surveys of the Mannheim Innovation Panel Conducted in the Years 2013 to 2016. 


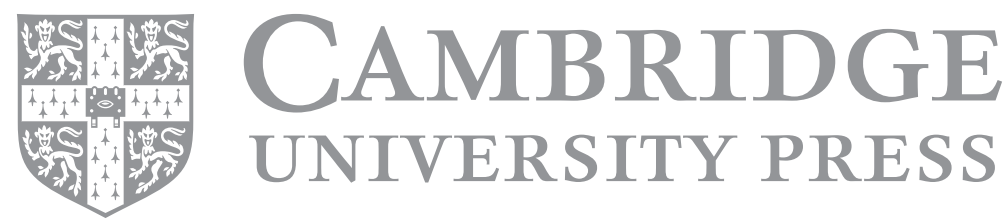

